\title{
Imaging Biological Function Across Scales: From Macromolecules to Cells to Microbial Communities
}

\author{
M. Auer* \\ * Lawrence Berkeley National Laboratory and Joint Bio-Energy Institute, 1 Cyclotron Road, \\ Mailstop 001, Berkeley, CA 94720
}

Over the last decade we have come to realize that biological function in cells is the result of spatial and temporal organization of macromolecular machines, allowing self-regulated complex metabolic and signaling networks to sustain life. And while we now easily accept such complex spatial and temporal organization for cellular function, it is less appreciated that such organization is not restricted to protein complexes but also occurs at the cell community level, e.g. in tissues and microbial communities/biofilms. Yet, such spatial and temporal 3D organization and cell-to-cell communication are much less well studied, although they are clearly essential for tissue and community integrity, and their respective physiological function.

Drastic improvement in biological sample preparation, allowing millisecond immobilization of biological processes and therefore faithful preservation, combined with advances in and development of novel imaging approaches have made it possible to image biological function across different scales, and hence to tackle different levels of complexity using the same specimen, by sequentially using different imaging modalities in a correlative manner. Since no single imaging modality can visualize the entire spectrum of scales and complexities, in fact it is imperative to utilize and integrate different imaging technologies in a correlative manner in order to gain more comprehensive biological insight.

Using examples from ongoing research in my laboratory I will illustrate the advantages and challenges of multiscale, multimodal imaging, show analyses both at the macromolecular complex as well as cell community level by using two types of examples:

(1) In branching morphogenesis of the mammary gland (and in a related study in-vitro models of breast cancer), we found the overall tissue organization to be preserved during the process of development while at the same time subcellular polarity breaking down, resulting in severely altered, ultrastructurally distinct cell-cell adhesion patterns. Fluorescence video microscopy allows the establishment of a history of the specimen and reveals its dynamic behavior, which can then be stopped at appropriate time point and examined at high resolution to examine macromolecular mechanisms that account for the behavioral phenotype. Interestingly, we found ultrastructural similarities between the process of mammary gland development and breast cancer progression.

(2) We have extensively studied microbial communities, both from soil bacteria as well as in the lignocellulose-degrading termite hindgut. I will show that such communities are exquisitely organized, and possess largely unstudied means of cell-to-cell communication and material exchange. For more complex communities organization exists beyond a simple stratification and most certainly reflects the respective macromolecular inventory. Since bacteria in mixed communities may be insufficiently distinct to allow mapping based on their morphological characteristics alone, our lab has been developing 16S rRNA-based affinity probe that allow correlative FISH/EM labeling in order to spatially map out community member distribution. FISH analysis establishes the relative position of species, whereas wide-field 2D TEM and 3D FIB/SEM 
imaging then can provide insight into the macromolecular inventory of metal reduction and/or lignocellulolytic biomass degradation, including extracellular cell appendages and secreted vesicles.

In addition, this presentation will highlight the extraordinary power of focused ion beam scanning electron microscopy (FIB/SEM) for 3D imaging of such complex microbial communities, if highcontrast resin-embedded samples, can be obtained. The level of detail that one can obtain from FIB/SEM imaging nearly rivals what can be accomplished only by electron tomography, but unlike electron tomography allows continuous imaging of tens of microns of specimen depth. 